\title{
Urgences
}

\section{Play ball : les neuf manches du temps et le champ de rêves de la fiction}

\section{Renald Bérubé}

Numéro 34, décembre 1991

Mythes et Romans de l'Amérique

URI : https://id.erudit.org/iderudit/025688ar

DOI : https://doi.org/10.7202/025688ar

Aller au sommaire du numéro

Éditeur(s)

Urgences

ISSN

0226-9554 (imprimé)

1927-3924 (numérique)

Découvrir la revue

Citer cet article

Bérubé, R. (1991). Play ball : les neuf manches du temps et le champ de rêves de la fiction. Urgences, (34). https://doi.org/10.7202/025688ar d'utilisation que vous pouvez consulter en ligne.

https://apropos.erudit.org/fr/usagers/politique-dutilisation/ 


\section{Play ball: les neuf manches du temps et le champ de rêves de la fiction Renald Bérubé}

\begin{abstract}
Aux membres de l'équipe de balle-molle de mon adolescence, celle de l'Auberge du Lac, en Salmonio
\end{abstract}

S'absorber dans ces jeux, c'était sentir son esprit tendre vers un espace de pure forme. En dépit de l'agitation qui régnait sur le terrain, le base-ball lui apparaissait comme une image de ce qui ne bouge pas, comme un lieu par conséquent où sa conscience pouvait trouver le repos et la sécurité, à l'abri des caprices de l'existence.

Paul Auster, L'invention de la solitude'

Gatsby hésita, puis ajouta froidement: * C'est lui qui a truqué le match international de baseball en $1919 \%$.

\section{F. Scott Fitzgerald, Gatsby le magnifique ${ }^{2}$}

« God what an outfield, " he says. "What a left field." He looks up at me and I look

\footnotetext{
I Paul Auster, L'invention de la solitude (traduit de l'américain par Christine Le Bcouf), Arles, Actes Sud, 1990, p. 142. Le texte original se lit ainsi: * To immerse himself in these games was to feel his mind striving to enter a place of pure form. Despite the agitation on the field, baseball offered itself to him as an image of that which does not move, and therefore a place where his mind could be at rest, secure in its refuge against the mutabilities of the world. "(The Invention of Solitude [1982], New York, Penguin Books, 1988, p. 115.)

2 F. Scott Fitzgerald, Gatsby $k$ magnifique, Paris, le Livre de poche, n* 900 , 1962, p. 114. Le texte original se lit ainsi : Gatsby hesitated, then added cooly: "He's the man who fixed the World's Series back in 1919." "(The Great Gatsby [1925], coll. "Contemporary Classics ", New York, Charles Scribner's Sons, [s. d.], p. 74.)
} 
down at him. * This must be heaven, " he says.

W.P. Kinsella,

Shoeless $J_{00}{ }^{3}$

Le * roman de base-ball o est si abondant, aux États-Unis, qu'il constitue un sousgenre, à l'instar du roman policier, du roman sentimental, ou du * western *.

Marc Chénetier, Au-delà du soup̧̧on ${ }^{4}$

Le baseball est, aux États-Unis, the national pastime. Non pas le sport national, ce qu'il devient le plus souvent en traduction française, mais bien the national pastime. Je lis, dans The Dickson Baseball Dictionary, à l'entrée national pastime: " $n$. A term commonly applied to baseball in the United States. First used in 1857, it eventually overshadowed other names such as national game and national sport. ${ }^{5}$ Cela étant, et parce que vous voulez assurer fermement la suite de votre argumentation, vous allez consulter votre Webster's New Collegiate Dictionary, édition de votre temps de collège justement, édition de 1956 plus précisément (1956: l'année où, comme tout un chacun sait, Don Larsen des Yankees de New York a lancé la seule et unique partie parfaite de l'histoire des Séries mondiales. Petit pleur vite réprimé aujourd'hui, mais non cette année-là : vous étiez partisan des Dodgers de Brooklyn, victimes de la perfection ponctuelle de Larsen) - vous allez donc consulter votre

3 W. P. Kinsella, Shoeless Joe [1982], New York, Ballantine Books, 1990, p. 16. La traduction pourrait se lire ainsi: " "Dieu quel champ extérieur", dit-il. "Quel champ gauche." II lève les yeux vers moi et je baisse les yeux vers lui. "Ce doit être le paradis", dit-il."

4 Marc Chénetier, Au-delà du soupçon (La nouvelle fiction américaine de 1960 à nos jours), coll. \& Le Don des langues n, Paris, Seuil, 1989, p. 150. Et si le " roman de baseball " est abondant aux États-Unis, il faut encore ajouter que bien des romans américains qui n'ont pas le baseball comme sujet premier, tels le Gatsby de Fitzgerald dont nous reparlerons ou Le vieil homme et la mer (1952) de Hemingway, se servent de cette pratique pour concrétiser ou métaphoriser leur propos.

5 Paul Dickson (edited and compiled by), The Dickson Baseball Dictionary, New York and Oxford, Facts on File, 1989, p. 271. 
Webster's au vocable pastime: " $n$. [pass + time] That which amuses or makes time pass agreeably; diversion; recreation ».

Un passe-temps ( $\times n$. m. inv. Occupation légère et agréable; divertissement ", selon le Petit Larousse illustré de 1973 - vous étiez alors professeur de littérature à l'UQAM et les moustachus A's d'Oakland allaient battre les Mets de New York dans les Séries mondiales de cette automne-là, en dépit des exploits de l'ex-Grand Orange des Expos, Rusty Staub, qui jouait même s'il était blessé), un passe-temps donc, tout national qu'il soit, n'exige pas la même crispation dans l'identification qu'un sport national. Puisque, par définition, un passe-temps, tout national qu'il..., demeure précisément cela, soit un passe-temps, une "occupation" qui "makes time pass agreeably".

Pour être bien clair: vous vous souvenez de l'extrême tension qui, au Canada et au Québec, fut notre lot collectif lors de la première confrontation (première * série du siècle ", bien sûr) entre nos meilleurs hockeyeurs et ceux de l'URSS en 1972? Le hockey est notre sport national, au Québec comme au Canada - et je sais bien, comme vous, que ni le Québec ni le Canada ne comptent beaucoup, contrairement aux États-Unis, de réalisations historiques sur la scène internationale. Nous n'allions pas de surcroît, menace intime à l'identité mal assurée, nous laisser battre à notre sport national! J'oserais dire que seules les trente-quatre dernières secondes, celles qui suivirent le but gagnant de Paul Henderson dans le huitième et dernier match de la série, passèrent agreeably. Et encore: il peut survenir tant de choses en trente-quatre secondes dans le temps particulier du hockey! Dernière remarque pour clore ce paragraphe: les Américains, alors même qu'ils n'évoluent pas sur la scène du monde, internationalisent néanmoins leurs pratiques: les Séries dites mondiales du baseball, n'ont pourtant été, jusqu'ici, jouées qu'aux États-Unis, entre clubs américains, même si la radio et la télévision les diffusent un peu partout sur la planète. Et vive l'ego serein et détendu. Distendu?

National pastime: la première occurrence de l'expression remonte à 1857, à une époque où la Guerre de Sécession n'avait pas encore eu lieu, et alors que les vastes territoires encore vierges, à l'ouest, pouvaient laisser croire qu'il y avait bien là, à l'infini et pour l'éternité ou presque selon l'american 
dream, un endroit dont chacun et chacune pouvait faire son espace personnel, son champ et son home. Un demi-siècle plus tard, au moment des premières Séries mondiales (1903) disons, le temps américain aura été à jamais marqué par la Guerre civile (1861-1865) et son espace aura dû reconnaître qu'une frontier ne peut être éternellement mouvante: le Pacifique a été atteint, limite ultime de la marche vers l'ouest. Une certaine innocence originelle a disparu, l'Histoire dorénavant réclame aussi ses droits. Et pourtant, le baseball demeurera pastime national, game (première occurrence: 1865) ou sport n'arrivant pas à supplanter la première appellation; comme si cette appellation originelle connotait des valeurs dont le baseball, bon gré mal gré et à son insu ou non, se trouvait porteur, des valeurs qui elles-mêmes traduisent à leur façon l'esprit national premier. National pastime.

Un passe-temps, donc, un passe-temps national: la notion de temps se révèle d'elle-même ici incontournable. Or il faut bien souligner, sans trop entrer dans les détails malgré la tentation, que le baseball est l'un des rares sports d'équipe, sinon le seul, qui regroupe les deux caractéristiques suivantes: 1) un match de baseball n'a pas un temps, une durée qui lui soit propre; son temps, sa durée s'inscrivent dans le temps réel, quotidien. Alors que les matches de hockey, de football (américain) ou de basketball durent une heure de leur temps, cette heure pouvant correspondre à trois heures environ de temps réel, les neuf manches réglementaires d'un match de baseball prennent, pour se dérouler, le temps qui leur est nécessaire. Le cadran ou la grande horloge qui égrène les minutes et les secondes, nouvel avatar du Temps impitoyable, n'a ici aucune importance, sinon celle de tous les jours, de tous nos jours dont nous ne savons pas quel sera leur nombre; 2) dans un match de baseball, l'un ou l'autre des deux clubs en présence ne peut marquer des points à n'importe quel moment. Au baseball, il est un... temps pour marquer des points: lorsque, dans une manche donnée, arrive votre tour au bâton (dans la première ou la seconde moitié de cette manche selon que vous jouez sur le terrain de l'autre ou chez vous), et un... temps pour empêcher l'adversaire d'en marquer: lorsque, dans une manche donnée, c'est votre tour d'être au champ, to take the field, selon l'expression rituelle. Si bien que, votre équipe étant au bâton alors qu'elle jouit 
déjà d'une avance dans le score, rien de (trop) malheureux ne peut advenir, elle ne peut toujours qu'ajouter à son avance, time pass[es] agreeably; si bien que, votre équipe jouant sur son terrain et étant en avance, elle n'aura même pas à venir au bâton dans la seconde demie de la neuvième manche, puisque sa victoire est déjà assurée. Quel autre sport peut se vanter de prendre ainsi ses aises avec le temps?

A quoi il faut ajouter, le temps ayant eu sa quote-part, que l'espace du match de baseball a aussi un intérêt certain. Puisque le mot field, champ, est ici le sémème intégrateur: il y a l'infield ${ }^{6}$, avant-champ ou champ intérieur dont les limites sont fermement établies, semblables en tout lieu où se déroule un match, et l'outfield ou champ extérieur (jouer dans l'outfield, comme voltigeur de gauche, de centre ou de droite, se dit aussi "jouer au champ " ou "jouer à la vache", en québécois) dont les configurations et les espaces peuvent varier beaucoup selon les stades où se déroulent les matches. Les caprices ou particularités d'un stade, issu(e)s de la géographie, de l'histoire, de l'économie ou que sais-je encore d'une ville, ont dans l'outfield bien des droits; joué d'abord dans le champ (ou presque) et en plein air, tels jadis les théâtres grec et élizabéthain, le baseball, la planète se rapetissant et l'économie des économistes et autres comptables réclamant son dû, a... dû suivre depuis quelques années la tendance à l'uniformisation et à la rentabilité. Si bien qu'il est aujourd'hui des stades couverts et des terrains synthétiques de plus en plus nombreux dont les dimensions du champ extérieur ont aussi tendance à se ressembler - mais en dépit de tout, le baseball demeure, aux États-Unis, le national pastime. Comme en 1857.

Littéraire impénitent, même lecteur, qu'il s'agisse d'un roman ou d'un match de baseball puisqu'il s'agit dans les deux cas d'un aménagement particulier du temps et de l'es-

6 A cause de sa forme, l'infield, avant-champ ou champ intérieur, est aussi appelé diamond, diamant. Ce qui explique le merveilleux titre polysémique (et à saveur bondienne), Diamonds Are Forever (Artists and Writers on Baseball). donné a une exposition itinérante sur le baseball; cette exposition avait été organisé par le New York State Museum en collaboration avec le Smithsonian Institution Traveling Exhibition Service. Le catalogue de l'exposition, que mon collègue Yves Lacroix de l'UQAM a eu la gentillesse de m'offrir, a été publié chez Chronicle Books, à San Francisco, en 1987. 
pace, lecteur impénitent qui utilise sa lecture de Roman des origines et origines $d u$ roman de Marthe Robert ${ }^{7}$ ou de Lire le théâtre d'Anne Ubersfeld 8 tout autant pour * lire * la littérature que pour "lire " un match de baseball, nous émettons l'hypothèse suivante: que le baseball demeure, aux ÉtatsUnis, le national pastime parce que sa pratique, malgré quelques changements ponctuels secondaires, continue d'incarner l'innocence des origines de la nation.

$$
* * *
$$

Il était une fois et il est encore, puisque vous l'avez vu pour une nième fois la semaine dernière, le film The Field of Dreams (Champ de rêves, en français) mettant en vedette Kevin Costner. Vous savez deux ou trois choses au moins s'agissant de ce film: 1) que Costner, avant de jouer dans Champ de rêves, avait joué dans La belle et le vétéran (Bull Durham, en anglais), film dont le baseball était aussi le sujet; 2) que le même Costner, après avoir joué dans Champ de rêves, allait réaliser (et jouer dans) Il danse avec les loups qui se déroule dans la prairie américaine du temps des «rencontres " Indiens-Blancs; 3) que Champ de rêves a été tourné à partir du roman de W. P. Kinsella intitulé Shoeless Joe.

Au moment où vous entreprenez d'analyser à la fois Shoeless Joe et Champ de rêves, vous savez qu'il vous faut au

7. Marthe Robert, Roman des origines et origines du roman [1972], coll. * Tel ", Paris, Gallimard, 1976. Peut-être est-ce lieu de citer ce qu'écrit Marc Chénetier qui résume alors un point de vue du romancier Robert Coover, auteur entre autres de Whatever Happened to Gloomy Gus of the Chicago Bears (traduit en français au Seuil sous le titre Une éducation en (liinois): « Les métaphores sportives, pour Coover, permettent de sonder les structures et les mentalités d'une nation à certains stades [!] de son développement. Le base-ball est ainsi à ses yeuX pertinent pour une considération globale du XIX $\mathrm{X}^{\bullet}$ siecle alors que le football - européen ou américain - renvoie au XX." (Au-dela du soupcon, p. 149).

8. Anne Ubersfeld, Lire le théâtre [1977], coll. * Essentiel ", Paris, Editions sociales, 1982. Si l'on accepte la formule de Paul Auster, à savoir que le baseball peut se lire comme * un espace de pure forme", on comprendra que le modèle actantiel greimassien et autres procédés d'enalyse structuro-sémiotique utilisés par Ubersfeld pour *lire le théâtre", puisse être utilisé pour lire le spectacle du baseball - ou mème des sports en général. D'accord? 
moins évoquer Palimpsestes ${ }^{9}$ de Gérard Genette, puisque le film est bien une adaptation du roman et qu'il relève en ce sens des pratiques hypertextuelles. Si, en gros, le film et le roman visent une même quête: réhabiliter la mémoire de Shoeless Joe Jackson, héros du père du narrateur, le film supprime cependant quelques personnages du roman (Richard Kinsella, le frère jumeau du narrateur; Eddie (Kid) Scissons qui fabule et raconte sa carrière fabulée de lanceur avec les Cubs de Chicago) et en transforme singulièrement un autre: le Terence Mann du film, écrivain noir fictif (et fétiche) des années 1960 , s'appelle dans le roman J. D. Salinger, auteur du très reel The Catcher in the Rye (1951) qui fut le roman fétiche de la jeunesse des années 50 . Si bien que là où le film célèbre les années 60 , le roman célèbre les années 50 - décennies fort différentes, on le sait. Et faut-il insister sur le fait que le père de Ray Kinsella, le narrateur du roman, jouait/joue au baseball au poste de receveur (catcher) et que Ray construit son stade dans un champ de maïs s'il ne le construit pas dans un champ de seigle (rye)? Ou sur le fait que le nom de Richard Kinsella, personnage qui ressemble comme un double à Ray son frère jumeau dans Shoeless Joe, se trouve effectivement dans Catcher in the Rye ${ }^{10}$ ainsi que le narrateur le fait remarquer à Salinger? Vous vous faites alors les remarques suivantes, palimpsestueusement: l'auteur (mal connu) d'un roman très réel devient donc personnage de roman; le narrateur de ce dernier roman a comme patronyme Kinsella, le même que celui de l'auteur dudit roman, et ce patronyme est encore celui d'un personnage du Catcher in the Rye de Salinger, personnage qui est le jumeau/ double du narrateur de Shoeless Joe - c'est clair? L'hypertextualité, décidément, dispose de toutes les astuces, permet toutes les gémellisations entre réalité et fiction; entre sport et littérature comme entre temps passé et temps présent aussi.

9 Gérard Genette, Palimpsestes (La Littérature au second degré), coll. "Poótique ", Paris, Seuil, 1982.

10 J. D. Salinger, The Catcher in the Aye [1951], New York, Bantam Book, 1964, p. 183. La traduction française de ce roman par Jean-Baptiste Rossi [Sébastien Japrisot!], L'attrape-ccours ( coll. « Pavillons ", Paris, Robert Laffont,1953) réserve au moins une grosse surprise au lecteur hypertextuel: le Richard Kinsella du Catcher devient Richard Kinsalla dans L'attrape-coeurs (p. 218). Le titre du roman de Salinger provient de la transformation par le héros de l'couvre, Holden Caulfield, d'un poème de Robert Burns (Catcher, p. 173). 
Retenir d'abord, littéraire et amateur de baseball que vous êtes, ce passage de Shoeless Joe que vous auriez voulu placer en épigraphe du texte que vous êtes en train d'écrire. Sa longueur vous a empêché de le faire, d'autant plus que ce passage ne constitue toujours qu'un bref extrait d'une longue discussion entre un narrateur (de roman de baseball)/lecteur (des romans de Salinger et du baseball), Ray Kinsella, et un auteur (de romans)/amateur de baseball, J. D. Salinger, sur la lecture possible et de l'œuvre littéraire et de la pratique du baseball. Le passage est le suivant où Ray s'adresse à Salinger:

You've captured the experience of growing up in America, the same way Freddy Patek corners a ground ball. The Catcher in the Rye is the definitive novel of a young man's growing pains, of growing up in pain. Growing up is a ritual - more deadly than religion, more complicated than baseball, for there seems to be no rules. Everything is experienced for the first time. But baseball can soothe even those pains, for it is stable and permanent, steady as a grandfather dozing in a wicker chair on a verandah (p. 72). ${ }^{11}$

Jeu de baseball, jeu de l'écriture: dans un cas comme dans l'autre, il s'agit d'apprendre et de grandir, de découvrir du neuf tout en étant assuré d'une certaine permanence. Le baseball et la littérature se jouent/s'écrivent à la fois dans le temps et au-delà de celui-ci. Les joueurs de baseball qui évoluent avec Shoeless Joe sur le terrain construit par Ray (dans ses champs de maïs de l'Iowa, au mépris des réalités économiques et des rires de ses voisins, la Voix l'ayant aisément emporté, pour Ray et son épouse, sur les \$) sont morts depuis plus ou moins longtemps; ils ne sont visibles qu'à ceux et

11 Commencer par dire que Freddie (et non Freddy) Patek a joué au poste d'inter (arrêt-court) pour diverses équipes du baseball majeur; ensuite, risquer la traduction suivante de ce passage: * Vous avez saisi l'expérience qu'est grandir aux États-Unis de la même manière que Freddie Patek attrape une balle frappée au sol. L'attrape-ccours est le roman définitif des souffrances croissantes d'un jeune homme, de ce qu'est grandir dans la souffrance. Grandir est un rite - plus mortel que la religion, plus compliqué que le baseball, puisqu'il semble n'y avoir aucune règle. Toute chose est expérimentée pour la première fois. Mais le baseball peut apaiser mème ces souffrances, parce qu'il est stable et permanent, présent comme un grand-pére assoupi dans une chaise d'osier sur une véranda. " 
108

celles qui savent reconnaître, nolens volens, tout à la fois le sens de la pérennité et du rêve, c'est-à-dire le sens de la création et de l'aventure.

A la fin du film, Ray et son père se lancent des balles de baseball, father playing catch with son pour paraphraser le titre d'un ouvrage du poète américain Donald Hall ${ }^{12}$; mais alors le fils est plus âgé que le père, il joue avec un père qui ne l'a pas conçu encore, et qui est décédé depuis longtemps. De même, il faut prêter une attention particulière à cette autre scène où le docteur Graham soigne la petite fille de Ray; le docteur Graham, à ce moment-là, habite à peu près simultanément trois temps différents: celui du présent du roman, celui de la fin de sa vie alors qu'il était médecin, et celui de sa jeunesse alors qu'il avait rêvé de jouer au baseball ainsi qu'il le fait avec l'équipe de Shoeless Joe. National pastime, le baseball sait jouer du temps - par un « roman de baseball » interposé qui ne traduit toujours que l'esprit du baseball.

Si Shoeless Joe se déroule en 1979 , il renvoie à un événement historique traumatisant qui s'est déroulé en 1919. Cette année-là, les White Sox de Chicago, pour lesquels jouait Shoeless Joe Jackson, voltigeur de gauche et grande vedette de l'équipe, perdirent la Série mondiale aux mains des Reds de Cincinnati alors qu'ils étaient donnés largement favoris pour l'emporter. Une enquête allait suivre qui prouverait que cette Série mondiale avait été truquée - les White Sox de 1919 allaient devenir, pour la postérité, les Black Sox du scandale. Huit joueurs des White Sox, dont Shoeless Joe, allaient être en 1920 bannis à vie de la pratique du baseball professionnel. Et cela, s'agissant de Shoeless Joe, et ainsi que l'affirme hautement Ray Kinsella en reprenant les arguments de son père et des historiens du baseball, en dépit d'une performance impeccable du célèbre voltigeur de gauche. Fautil bien insister: réhabiliter le héros du père, c'est aussi retrouver le père et, donc, les origines. Les origines familiales, bien sûr, qui ne sont toujours que la métaphore ponctuelle, personnelle et intime, des Origines; en l'occurrence, celles du national pastime de 1857 et de la nation qui a ainsi nommé cette pratique.

12 Donald Hall, Fathers Playing Catch with Sons. Essays on Sport [Mostly Baseball], New York, Laurel Book, 1986. 
Il m'arrive souvent de croire, comme à vous sans doute, que Gatsby le magnifique de F. Scott Fitzgerald, publié en 1925 , est peut-être LE grand roman américain que tout romancier US qui se respecte rêve encore d'écrire ${ }^{13}$. D'une écriture merveilleuse de simplicité et de complexité, de finesse, dont s'est peut-être souvenu le Salinger du Catcher, admirablement structuré dans son déroulement par le récit d'un narrateur qui était l'ami de Gatsby et qui a donc participé à l'aventure qu'il raconte, le roman décrit à la fois l'american dream et sa perversion, la naïveté simple et romantique de ce rêve qui, dans le cas de Gatsby, ne peut pourtant se réaliser que si le héros emprunte des moyens qui sont l'antithèse de but visé. Comme si la perversion du rêve dans les moyens de la quête pouvait mener à la réalisation de celle-ci. Pour conquérir la riche Daisy, sorte de southern belle, Gatsby se fera bootlegger, métier qui rapporte vite et gros et qui en ferait un parti digne de la richesse qu'étalent Daisy et sa famille, mais métier qui demande qu'on n'hésite pas à sacrifier des vies humaines aux lois (!) de la réussite financière selon des pratiques illégales. Le paradoxe « lois (!) de la réussite/pratiques illégales »: il veut signifier que l'argent, symbole de réussite, est aussi agent du détournement du rêve. Gatsby perdra tout: son amoureuse, son rêve, sa vie. Le héros éponyme, formé par un dénommé Cody dont le patronyme n'est pas sans rappeler celui de Buffalo Bill (William Cody) et donc l'aventure de la prairie américaine, aura aussi eu Meyer Wolfsheim comme maître, celui-là même qui a organisé la tricherie de la Série mondiale de 1919.

13 II vaut la peine de souligner que Philip Roth a publié en 1973 un roman qui porte préciserment ce titre, The Great American Novel, et qui relate les aventures d'une équipe de baseball. Roth, on le sait, avait déjà consacré quelques belles pages au baseball dans Portnoy's Complaint publié en 1969, notamment ces pages où Portnoy se rêve en Duke Snider, très rélle vedette des Dodgers de Brooklyn durant les années cinquante et soixante. The Great American Novel a été traduit en français par Sylvie Salade: Le grand roman americain, coll. « Du monde entier ", Paris, Gallimard, 1980. II y aurait long à dire sur cette traduction, sur les explications du jeu de baseball qui la précedent et sur le glossaire final qui l'accompagne; il est d'autant plus difficile de traduire que cela méme qui est traduit constitue une pratique mal connue. Cela dit, le roman de Roth a été traduit, mème si la traduction a mis du temps à venir; quand donc sera traduit The Natural publie par Bernard Malamud on 1952 ? Mais comment donc traduire correctement le simple titre de ce roman, The Netural? 
110

Au cour de Gatsby, telle une métaphore en abyme, la Série mondiale truquée de 1919: le rêve et sa dégénérescence. Malgré tout, le rêve reste le plus fort: Gatsby demeure un héros de la frontière, amoureuse ou autre, son caractère hors-la-loi n'étant toujours qu'une concession momentanée et obligée à la fois à l'ampleur du rêve (qui doit durer) et aux lois (!) de la réussite (mal nécessaire). Mais la dégradation du rêve est là, soulignée: qui dit que le mal nécessaire ne l'emportera pas, toujours et finalement, sur le rêve pérenne? Discréditant la sanction imposée à Shoeless Joe, W. P. et Ray Kinsella disent que celle-ci ne visait qu'à sauver la crédibilité de l'industrie du baseball. Alors que la Justice aurait dû tenir compte de l'innocence/naïveté de Shoeless Joe: son pseudonyme, Shoeless, disant déjà son contact primitif avec la nature, et sa performance de rêve dans la Série de 1919 témoignant irrévocablement en sa faveur. L'esprit ou la magie du baseball - et Shoeless Joe n'hésite pas à recourir aux moyens des contes de fée ou du fantastique - ne doivent pas céder le pas à l'industrie mercantile du national pastime; il faut ce qu'il faut pour empêcher l'art et le rêve de succomber aux lois du marché.

\section{***}

L'américanité, le Québec, le Canada, les États-Unis. Et la littérature, puisque la revue où doit paraître ce texte est une revue littéraire de théorie et de création, revue dont vous êtes membre du comité de rédaction. À ce moment-ci où vous êtes à la veille de conclure, ajouter ce petit détail innocent que vous avez jusqu'ici, sans aucune innocence, omis de souligner: Shoeless Joe n'est pas l'œuvre d'un romancier américain mais bien d'un auteur canadien (albertain) né en 1935. Voilà. La mémoire et le pastime ayant leurs droits, vous vous souvenez alors de cet article d'un autre auteur de l'Ouest canadien, George Bowering, article intitulé * Le baseball et l'imaginaire canadien $* 14$ et dont deux des sous-titres étaient particulièrement plaisants: "Octosyllabes et neuf manches ",

14 George Bowering, "Le baseball et l'imaginaire canadien ", Nuit blanche, $n^{\circ} 29$ (numéro intitulé Le sport a des lettres), octobre-novembre 1987, p. 44-48. 
«Le baseball sied aux poètes *. Thèse de Bowering: «Ce n'est pas le hockey qui est le plus intimement lié à l'imaginaire canadien, mais le baseball. $»$ J'avoue mon hésitation à pousser si loin l'affirmation, même si je me dis que pour lui comme pour moi l'automne ne doit réellement commencer qu'au moment du début des Séries mondiales; et je suis prêt à admettre que le baseball occupe, dans la littérature américaine, une place que n'occupe pas (encore) le hockey dans les littératures canadienne et québécoise. Même que Guillaume Plouffe, dans le roman de Roger Lemelin ${ }^{15}$, se destinait à une carrière dans le baseball; c'est la série radiophonique tirée de ce roman qui en fera un joueur de hockey, ô mystères de l'hypertextualité ou du public destinataire.

Mais il faut être d'accord avec Marc Chénetier quand il associe "roman[s] de baseball * et " roman[s] western * - en ajoutant par ailleurs qu'il s'agit moins ici, pour nous, du roman western que de la musique country / western qui a, au Québec, de profondes racines: le baseball et cette musique, chaque pratique selon sa façon, incarnent bien la nostalgie immémoriale des origines, du temps simple et rêvé (fantasmé) des origines. "Le passé du baseball professionnel est intact ", écrit le narrateur dans la seconde partie de L'inven. tion de la solitude de Paul Auster 16; et à son grand-père à la veille de mourir, il fait lecture, à la demande du grand-père, des résultats des matches de baseball. Toujours « pareil à luimême * ${ }^{17}$, le baseball sait tout autant marquer le passage du temps que lier les générations.

15 Roger Lemelin, Les Plouffe [1948], Montréal, La Presse, 1973. * En attendant, ménage ton bras. Les grandes ligues de baseball des États t'attendent m, écrit Napoléon à son jeune frère alors à la guerre (p. 383).

16 Paul Auster, L'invention de la solitude, p. 144. C'est l'innocence et la paix, la * gentility of the past " que viendront chercher les spectateurs et spectatrices à ton stade, dit à peu prés Salinger à Ray (Shoeless Joe, p. 212).

17 Ibid., p. 143. Et il ne saurait être question de terminer ces notes sans mentionner deux ouvrages récents sur le baseball écrits par des intellectuels américains, ce qui dit assez la fascination que ce sport exerce, national pastime: du columnist George F. Will, Men at Work (The Craft of Baseball), New York, Harper Perennial, 1991 - ouvrage fascinant sur la finesse qu'exige la pratique du baseball, ouvrage plein d'humour aussi qui contient, à la fin, une brève analyse du film Bull Durham; et de l'essayiste David Halberstam, Summer of 49, roconstitution minutieuse et amoureuse, sorte de mémoires personnels et collectifs tout à la fois, de la lutte au championnat de la Ligue américaine que se livrèrent cet été-là les Yankees de New York et les Red Sox de Boston. 
112

"The play is the thing *, disait Hamlet en parlant du théâtre; "to play ball is the pastime ", disent Kinsella, Roth et Auster eux aussi fascinés par la vérité et les origines comme par les dires du père. Ou du grand-père assoupi sur la véranda, si près du paradis. 